\title{
Order/Disorder Dynamics in a Dodecanethiol-Capped Gold Nanoparticles Supracrystal by Small-Angle Ultrafast Electron Diffraction
}

\author{
Giulia Fulvia Mancini, ${ }^{\dagger}$ Tatiana Latychevskaia, ${ }^{\dagger}$ Francesco Pennacchio, ${ }^{\dagger}$ Javier Reguera, ${ }^{\S}$ \\ Francesco Stellacci, ${ }^{\S}$ and Fabrizio Carbone* ${ }^{* \dagger}$
}

${ }^{\dagger}$ Laboratory for Ultrafast Microscopy and Electron Scattering, Lausanne Center for Ultrafast Science (LACUS), École Polytechnique Fédérale de Lausanne, CH-1015 Lausanne, Switzerland

${ }^{\ddagger}$ Physics Institute, University of Zurich, Winterthurerstrasse 190, 8057 Zurich, Switzerland

${ }^{\S}$ Supramolecular Nanomaterials and Interfaces Laboratory, Institute of Materials, École Polytechnique Fédérale de Lausanne, CH-1015 Lausanne, Switzerland

Supporting Information

ABSTRACT: The design and the characterization of functionalized gold nanoparticles supracrystals require atomically resolved information on both the metallic core and the external organic ligand shell. At present, there is no known approach to characterize simultaneously the static local order of the ligands and of the nanoparticles, nor their dynamical evolution. In this work, we apply femtosecond small-angle electron diffraction combined with angular cross-correlation analysis, to retrieve the local arrangement from nanometer to interatomic scales in glassy aggregates. With this technique we study a two-dimensional distribution of functionalized gold nanoparticles deposited on amorphous carbon. We show that the dodecanethiol ligand chains, coating the gold cores, order in a preferential orientation on the nanoparticle surface and throughout the supracrystal. Furthermore, we retrieve the dynamics of the supracrystal upon excitation with light and show that the positional disorder is induced by light pulses, while its overall homogeneity is surprisingly found to transiently increase. This new technique will enable the systematic investigation of the static and dynamical structural properties of nanoassembled materials containing light elements, relevant for several applications including signal processing and biology.

KEYWORDS: Angular cross-correlation function analysis, supracrystal, nanoparticles, electron diffraction, time-resolved

Tn metal nanoparticles (NPs), ${ }^{1-3}$ the metallic core provides some key properties, e.g., magnetization, plasmonic response, or conductivity, with the ligand molecules giving rise to others like solubility, assembly, or interaction with biomolecules. ${ }^{4,5}$ The formation of NPs supracrystals depends on a complex interplay between many forces, some stemming from the core, some from the ligands. ${ }^{6}$ These assemblies are promising candidates for many applications in very different fields, such as electronics and medicine. ${ }^{7,8}$

The structural dynamics of nonfunctionalized polycrystalline gold thin films has been investigated by means of ultrafast electron diffraction (UED) in transmission ${ }^{9,10}$ and timeresolved X-ray diffraction (XRD). ${ }^{11,12}$ Direct structural information on the melting and crystallization dynamics of gold NPs suspended on organic membranes was obtained by UED, ${ }^{13}$ while coherent acoustic phonons have been observed in single bare gold NPs by means of time-resolved threedimensional imaging in a X-ray free-electron laser (X-FEL). ${ }^{14}$
All of these experiments were limited at the observation of the intra-NP dynamics, and none dealt with functionalized NPs.

While electrons offer a higher cross-section for interaction with matter than $\mathrm{X}$-rays, yielding a good sensitivity in thin samples containing light elements, ${ }^{15,16}$ their limited transverse coherence complicates the observation of large distances in diffraction. ${ }^{17}$ Conversely, X-ray beams have the required coherence, but smaller interaction cross-section, making it difficult to observe the dynamics of the chains of carbon and hydrogen atoms constituting the ligands.

Structure retrieval methods using the angular crosscorrelation function (CCF) analysis of an XRD pattern have demonstrated the possibility to retrieve information on the sample local order and symmetry. ${ }^{18,19}$ Latychevskaia et al. ${ }^{20}$

Received: January 27, 2016

Revised: February 24, 2016

Published: February 26, 2016 


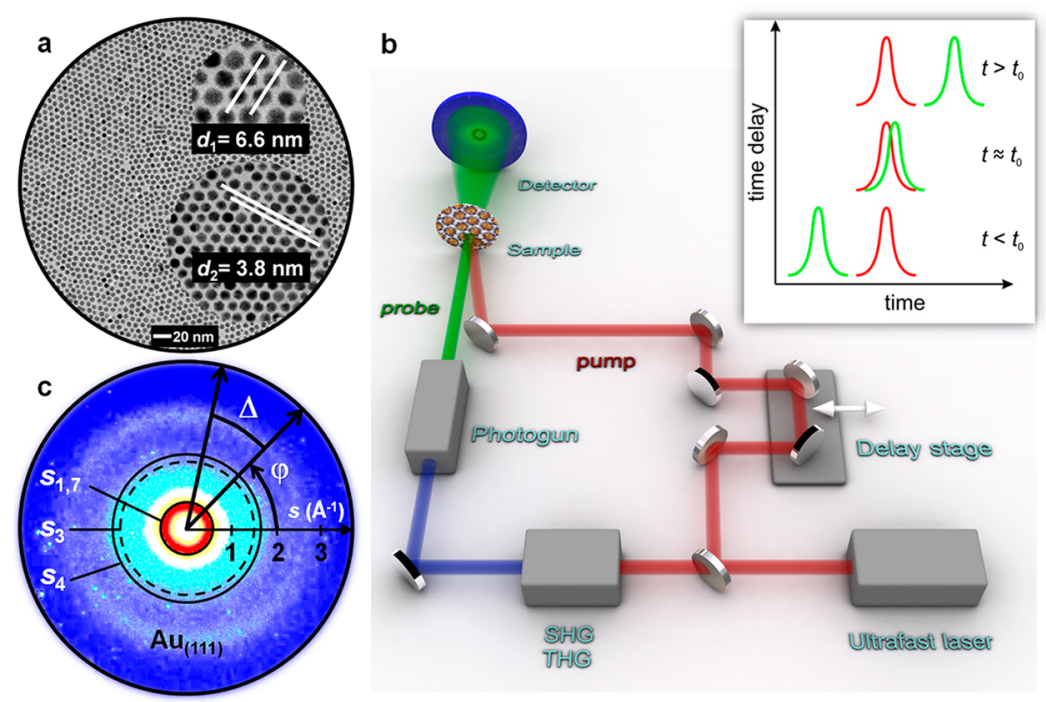

Figure 1. UED setup and principle for small-angle time-resolved electron diffraction. (a) TEM image of the sample in static conditions. Insets: Arrangement of the NPs and crystallographic planes with distances $d_{1}$ and $d_{2}$. (b) Schematic layout of the UED experiment. A $160 \mu \mathrm{m}$ diameter electron beam with partial coherence of $5 \mathrm{~nm}$ and $7 \mathrm{pm}$ wavelength is impinging on the sample which is at a distance of $23 \mathrm{~cm}$ from a charge coupled device camera with $47 \mu \mathrm{m}$ pixel size. For each time-delay 740 diffraction patterns containing $2 \times 10^{5}$ electrons each have been acquired and summed. Inset: The ultrashort light and electron pulses are arranged in a properly timed sequence with the use of a delay stage, and the sequence of pulses is repeated timing the electron pulse to arrive before or after the laser pulse. In this way snapshots of the electrons diffracted from the sample are recorded in a stroboscopic fashion. (c) Experimental electron diffraction pattern of the sample at $t<t_{0}$. The investigation of the local symmetry in our sample is carried out at the three marked scattering vectors $s_{1,7}$ (referred to as $s_{1}$ within the text), $s_{3}$ and $s_{4}$, as discussed in the text. The azimuthal angle $\varphi$ and the angle shift $\Delta$, employed in the calculation of the CCF, are also reported in the diffraction pattern.

reported that for a dense system of identical particles, even at a very low coherence length of the probing wave (comparable to the size of the single particle), the characteristic modulations of certain symmetries in the sample appear in the CCF.

Small-angle femtosecond (fs)-electron diffraction experiments presented in this work can access the length-scale between few Ångstrom ( $\mathrm{A})$ to a few nanometers (nm) with fs time-resolution and sensitivity to the light elements in ligands. To do this, a trade-off between the beam brightness and its coherence at the sample was found in our high-flux $\left(10^{9} \mathrm{e}^{-} / \mathrm{s}\right)$ UED setup. ${ }^{21}$ The CCF analysis has been applied and revealed the ordered arrangement of the ligands binding to the NPs in the two-dimensional supracrystal, even though the NPs themselves are not arranged in a perfect lattice. The discovered arrangement of the ligands indicates the presence of a specific preferred orientation of the NPs in the two-dimensional supracrystal. Furthermore, time-resolved experiments clarify the way in which light-induced thermal disorder evolves in such systems, revealing the time-scales involved in the entropy variations of the distribution of the NPs in the supracrystal and of the ligands sublattices.

Set-up and Experiment. 1-Dodecanethiol-coated NPs were synthesized using a modification ${ }^{22}$ of the method described by Zheng et al. ${ }^{23}$ A NPs monolayer was prepared by a dropwise deposition of a toluene solution of NPs onto the water subphase of a Langmuir trough. After $10 \mathrm{~min}$ the layer was compressed at $10 \mathrm{~mm} / \mathrm{min}$ until it reached $18 \mathrm{mN} / \mathrm{m}$ (at the isotherm solid-phase region), and then it was transferred to an amorphous carbon-coated grid through a LangmuirSchaefer deposition. A transmission electron microscopy (TEM) image of the sample, characterized by an average NPs core diameter of $5.7 \mathrm{~nm}$ and a polydispersity of $9 \%$, is shown in Figure 1a, where crystallographic-like planes in the NPs arrangement are indicated. In this TEM image, the ligands are not visible as they do not provide enough contrast for 200 $\mathrm{keV}$ electrons. Previous experiments demonstrated that the ligands may be observed at lower voltages ${ }^{24,25}$ for isolated particles. Recently, the ligands orientation on an ensemble of oleic-acid-coated $\mathrm{PbSe}$ nanoparticles has been imaged in a HAADF-STEM operated at $200 \mathrm{kV} .^{26}$ However, none of these experiments has provided, at once, static and dynamic information on ligands, as they were limited to the observation of the system in stationary conditions.

A KMLabs Wyvern Ti:sapphire amplified laser generating 50 fs (fwhm), $700 \mu \mathrm{J}$ pulses, $800 \mathrm{~nm}$ central wavelength, $20 \mathrm{kHz}$ repetition rate, was employed to generate both the probing electrons ( $30 \mathrm{keV}, \lambda=7 \mathrm{pm}$ ) and the photoexcitation. The temporal spread of the $30 \mathrm{kV}$ probe electron pulses was controlled by means of a radiofrequency (RF) compression cavity, ${ }^{27,28}$ allowing to store up to $6 \times 10^{5}$ electrons in $\sim 300 \mathrm{fs} /$ $160 \mu \mathrm{m}$ bunches at the sample. ${ }^{21}$ A sketch of our setup is presented in Figure 1b. Femtosecond snapshots of the diffraction pattern from the NPs supracrystal were recorded at different time-delays between the pump photoexcitation and the probe, building a femtosecond-resolved movie of the dynamics of the sample. Experiments were performed in transmission geometry at room temperature and with an almost collinear arrangement between the pump and probe pulses, in order to reduce the spatiotemporal mismatch between them. The background pressure in the experimental vacuum chamber was below $10^{-9}$ mbar. Photoinduced changes in the sample were initiated by $800 \mathrm{~nm}(1.5 \mathrm{eV})$ pump-pulses focused to a spot of $220 \mu \mathrm{m}$, which were temporally overlapped on the sample to a probing electron beam with $160 \mu \mathrm{m}$ diameter. The incident fluence on the sample was $10 \mathrm{~mJ} / \mathrm{cm}^{2}$, while the effective fluence absorbed by the sample is estimated around $100 \mu \mathrm{J} / \mathrm{cm}^{2}$. This latter takes into account for the optical reflectivity of gold in a layer of $7 \mathrm{~nm}$ thickness, for the 

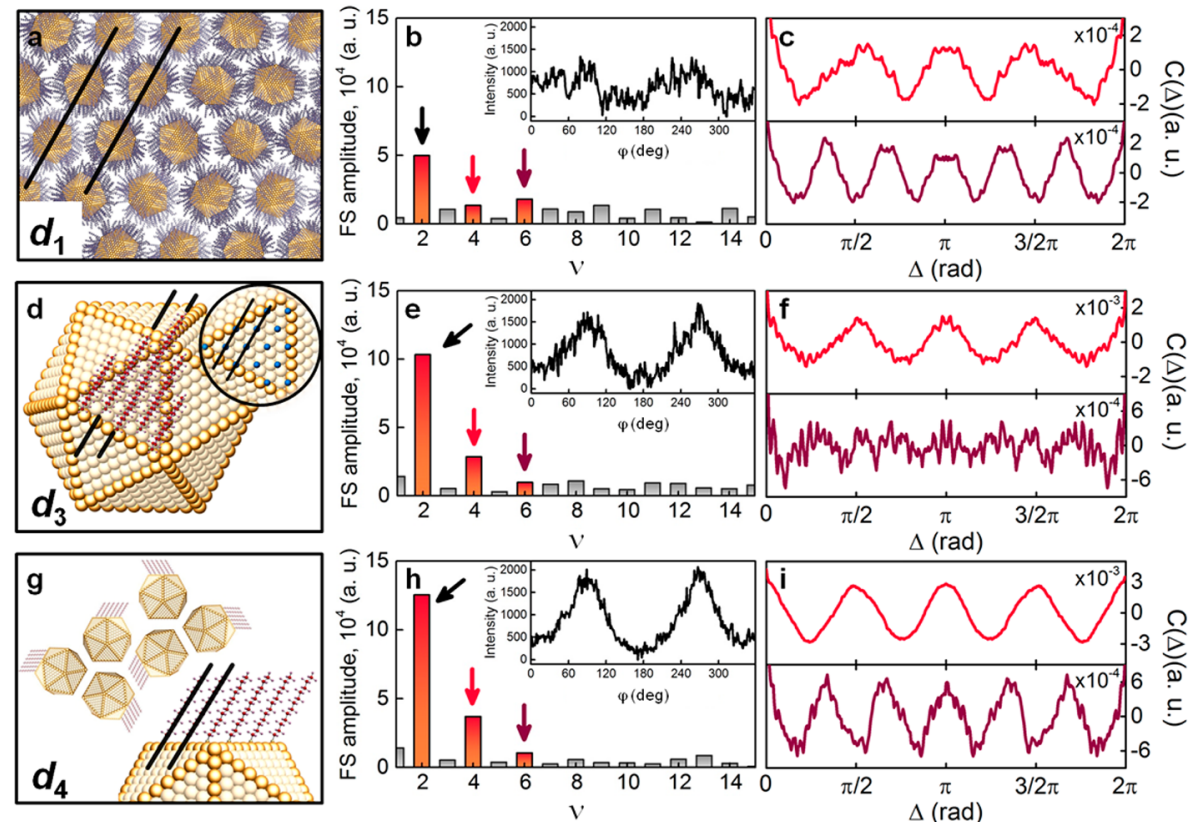

Figure 2. Static Fourier spectrum analysis at $d_{1}, d_{3}$, and $d_{4}$. (a, d, g) Rendering of the real space objects corresponding to the distances $d_{1}\left(s_{1}\right), d_{3}\left(s_{3}\right)$, and $d_{4}\left(s_{4}\right)$, respectively. (b, e, h) Fourier spectrum obtained by the Fourier transform of the angular intensity profile (insets, black curves). (c, f, i) Upper, red curves: CCF obtained by setting to zero the frequency $\nu=2$ in the Fourier spectrum. Lower, purple curves: CCF obtained by setting to zero the frequencies $\nu=2$ and $\nu=4$ in the Fourier spectrum. All of the Fourier spectra as well as the angular intensity curves show a $\nu=2$ frequency. This behavior is assigned to the astigmatism of the electron beam and the presence of amorphous carbon in the sample support, which is the subject of extended discussion in the SI. The CCF curves have been obtained from the raw data following dark noise subtraction and following the procedure explained in the text. The "large" values of the CCF are motivated by the presence of local ordering throughout the entire sample, as theoretically demonstrated in ref 20 .

penetration depth of gold for electrons, estimated around 7-8 $\mathrm{nm}$ at $1.5 \mathrm{eV},{ }^{29}$ and for the sample density.

The diffraction patterns were acquired with a very small current (320.4 pA for every time delay, $4.11 \mu \mathrm{C}$ as total charge on the NPs with this amount being distributed over several electron pulses impinging on the sample) thanks to the high sensitivity camera and the pulsed electron beam. Also, the very low duty cycle allows a large relaxation time between subsequent pulses. For these reasons, radiation damage was not observed in these experiments (see Supporting Information). The diffraction pattern, shown in Figure 1c, was formed on a phosphor screen and was recorded by a charge-coupled device camera capable of single electron detection; in the experiment, the sample-to-camera distance was optimized so that the transverse coherence was comparable to the distances of interest in the sample $(\sim 5 \mathrm{~nm})$, corresponding to small deflection angles in the mrad range.

Static Characterization of the Supracrystal. The investigation of the local symmetry in our sample is carried out at the three different scattering vectors marked in the diffraction pattern of Figure 1c, which relate to three different length scales in the sample. The low order feature $s_{1}$ is attributed to the arrangement of the NPs in the supracrystal, where the NPs form crystallographic planes with a distance $d_{1}=$ $6.6 \mathrm{~nm}$ as depicted in the upper inset of Figure 1a and sketched in the rendering of Figure $2 \mathrm{a}$.

The features at higher scattering vectors, labeled $s_{3}$ and $s_{4}$, correspond to the arrangement of the ligands binding to the surface of each gold NPs core. The reciprocal distance $s_{3}$ refers to the hexagonal superlattice of dodecanethiol ligands chains that are binding the gold core facets at specific locations between gold atoms, as shown in the inset of Figure $2 \mathrm{~b}$. In the gold face center cubic $(f c c)$ crystal structure, the distance between two neighboring gold atoms is $2.88 \AA$, which leads to a distance between two nearest locations of the ligand attachment of $4.99 \AA$ (see inset of Figure $2 \mathrm{~d}$ ). This gives the distance between the crystallographic planes created by the carbon chains of $d_{3}=\sqrt{3 / 2} \times 4.99 \AA=4.33 \AA$. A typical geometrical arrangement of the system in static conditions is the one in which the ligands attached to one facet are stretched and the NPs are randomly oriented throughout the sample, as explained in details in the Supporting Information (SI). In this situation, a diffraction ring at $s_{3}=1.45 \AA^{-1}$ is expected in the diffraction pattern.

The signal at the scattering vector $s_{4}=1.68 \AA^{-1}$ is related to the real space distance $d_{4}=3.72 \AA$, which is the distance between chains of carbon atoms in ordered ligands when those are projected from a specific direction of view, as depicted in Figure $2 \mathrm{~g}$. This orientation of ligands relates to a peculiar orientation on the NPs which turns out to be the preferred orientation of NPs throughout the sample, as discussed later in the text.

Cross-Correlation Function and Fourier Analysis of the Diffraction Pattern. Local symmetries in the sample can be revealed by analyzing the modulations in the CCFs at the related scattering vectors $s_{j}(j=1,3,4)$. The normalized CCF is defined as ${ }^{18,19}$

$$
C_{\text {norm }}(\Delta)=\frac{\langle I(s, \varphi) I(s, \varphi+\Delta)\rangle_{\varphi}-\langle I(s, \varphi)\rangle_{\varphi}^{2}}{\langle I(s, \varphi)\rangle_{\varphi}^{2}}
$$

where $I(s, \varphi)$ represents the scattered intensity at the defined scattering vector $s$ and the angle $\varphi ; \Delta$ is the shift between the two angles, as indicated in Figure $1 \mathrm{c}$ and \langle\rangle$_{\varphi}$ denotes an 

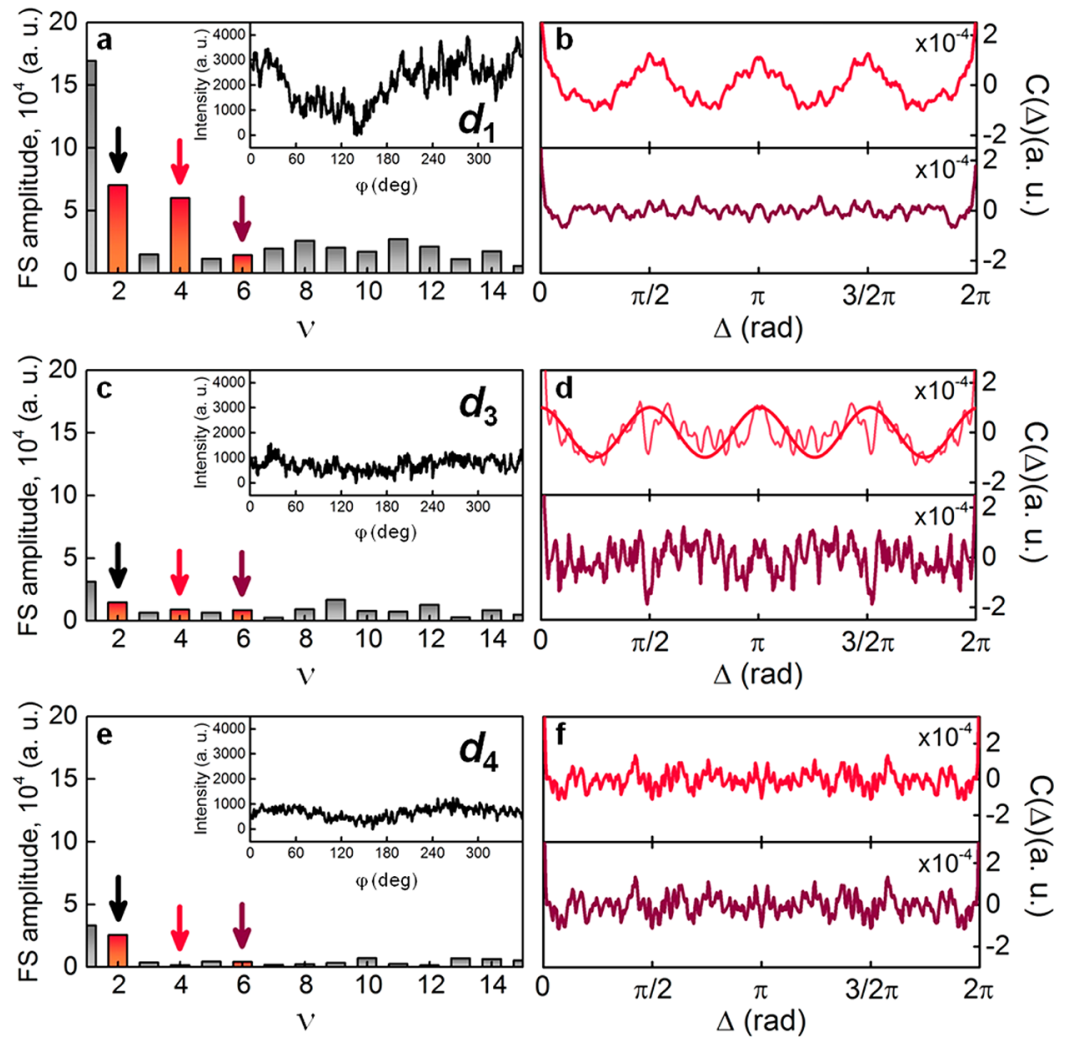

Figure 3. Fourier spectra and CCFs at $s_{1}, s_{3}$, and $s_{4}$ in the amorphous-carbon coated TEM grid. (a, c, e) Fourier spectra obtained by the Fourier transform of the angular intensity profile (insets, black curves). (b, d, f) Upper, red curves: CCF obtained by setting to zero the frequency $\nu=2$ in the Fourier spectra. Lower, purple curves: CCF obtained by setting to zero the frequencies $\nu=2$ and $\nu=4$ in the Fourier spectra. The data show clearly that the information retrieved from the supracrystal are different from the ones of the substrate.
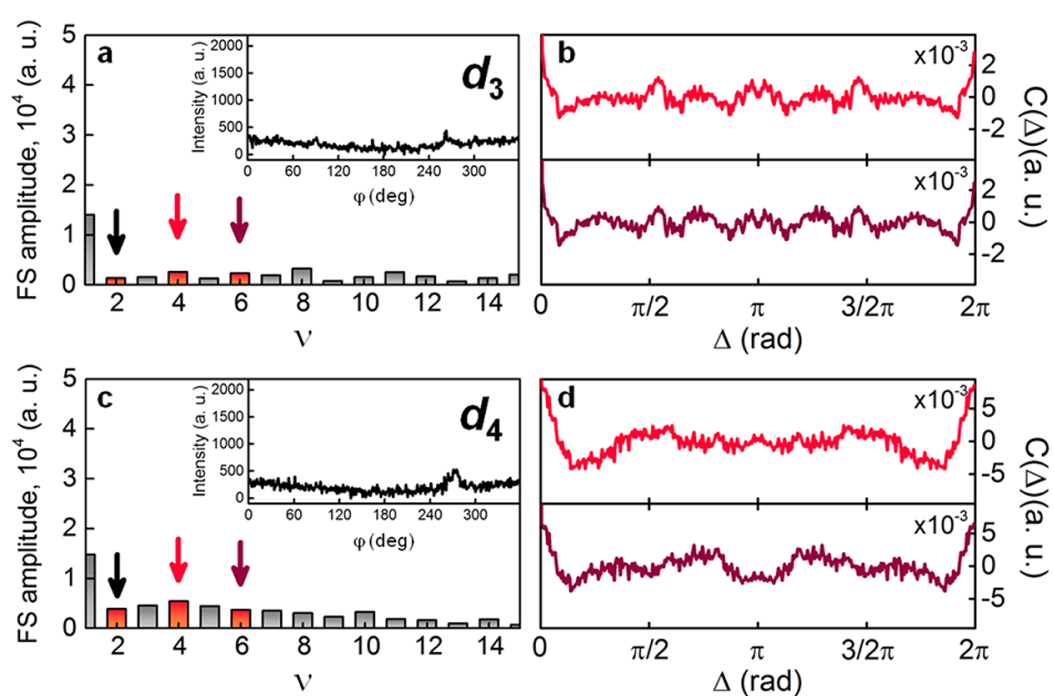

Figure 4. Fourier spectra and CCFs at $s_{3}$ and $s_{4}$ in the empty copper TEM grid. (a, c) Fourier spectra obtained by the Fourier transform of the angular intensity profile (insets, black curves). (b, d) Upper, red curves: CCF obtained by setting to zero the frequency $\nu=2$ in the Fourier spectra. Lower, purple curves: CCF obtained by setting to zero the frequencies $\nu=2$ and $\nu=4$ in the Fourier spectra. Also in this case, no specific symmetries are retrieved, as opposed to the sample. The data at $s_{1}$ are not shown because the beam through the empty grid oversaturates the detector at very small angle, preventing the analysis of the data.

averaging over $\varphi$. The details on the formalism of the angular cross-correlation analysis are reported elsewhere. ${ }^{30,31}$ The nonnormalized CCF is calculated as

$$
C(\Delta)=\langle I(s, \varphi) I(s, \varphi+\Delta)\rangle_{\varphi}=\operatorname{Re}\left(F_{\varphi}^{-1}\left(\left|F_{\varphi}\{I(s, \varphi)\}\right|^{2}\right)\right)
$$

where $F_{\varphi}\{\ldots\}$ is the one-dimensional Fourier Transform (FT) over the $\varphi$ coordinate. The Fourier transform of $I(s, \varphi)$ yields a spectrum where the most pronounced frequencies indicate the presence of a signal with a corresponding periodicity. In our data, the low-order frequency $\nu=2$ is assigned to the beam astigmatism and to the signal from the amorphous carbon 
Table 1. Symmetries Observed in the CCFs from the Experimental Diffraction Pattern Are Assigned to the Corresponding RealSpace Objects ${ }^{a}$

\begin{tabular}{|c|c|c|c|c|c|}
\hline & \multicolumn{2}{|l|}{ NP sample } & \multicolumn{2}{|l|}{ TEM grid + carbon } & $\begin{array}{l}\text { empty copper } \\
\text { TEM grid }\end{array}$ \\
\hline & 4-fold & 6-fold & 4-fold & 6 -fold & 4-fold 6-fold \\
\hline$S_{1}$ & $\begin{array}{l}\text { tetragonal arrangement of the amorphous } \\
\text { carbon in the substrate }\end{array}$ & $\begin{array}{l}\text { the supracrystal } \\
\text { structure }\end{array}$ & $\begin{array}{l}\text { tetragonal arrangement of the amorphous } \\
\text { carbon in the substrate }\end{array}$ & & \\
\hline $\begin{array}{l}S_{3} \text { random orientation } \\
\text { of NPs }\end{array}$ & $\begin{array}{l}\text { tetragonal arrangement of the amorphous } \\
\text { carbon in the substrate }\end{array}$ & & $\begin{array}{l}\text { tetragonal arrangement of the amorphous } \\
\text { carbon in the substrate }\end{array}$ & & \\
\hline $\begin{array}{l}S_{4} \text { preferred } \\
\text { orientation of NPs }\end{array}$ & NPs local arrangement in the supracrystal & $\begin{array}{l}\text { the supracrystal } \\
\text { structure }\end{array}$ & & & \\
\hline
\end{tabular}

${ }^{a_{T}}$ The modulations that are a signature of the NPs supracrystal are separated from the ones of the TEM grid supporting the supracrystal or the substrate.
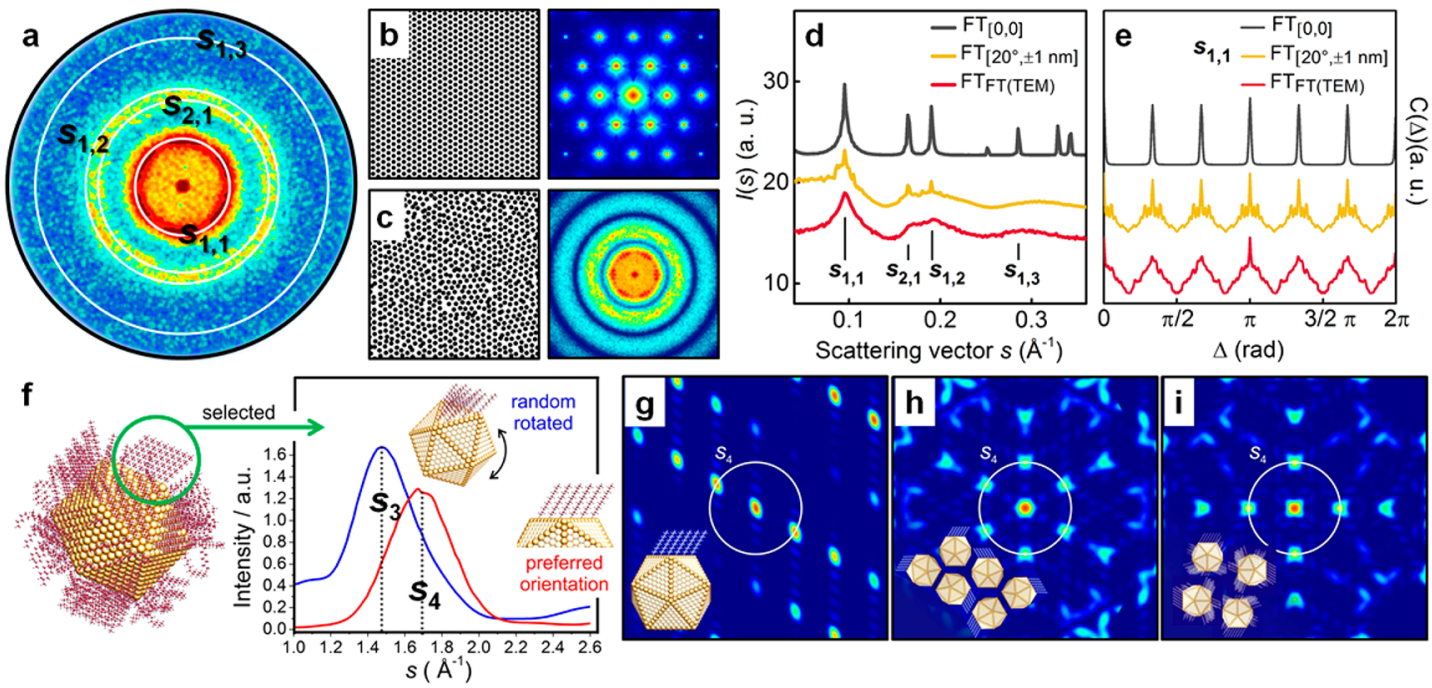

Figure 5. SLM and SASA simulations. (a) Squared amplitude of the Fourier transform of the experimental TEM image of the sample. Three different diffraction orders $n=1,2,3$ are detected from $s_{1}\left(d_{1}=6.6 \mathrm{~nm}\right)$, together with the first order $(n=1)$ from $s_{2}\left(d_{2}=3.8 \mathrm{~nm}\right)$. The corresponding crystallographic planes in the supracrystal are marked in Figure 1a, in the upper and lower insets, respectively. (b) NPs distribution simulated in SLM from a perfect lattice (domains rotation Gaussian distributed with mean $=0^{\circ}$, standard deviation $\sigma=0^{\circ}$, NPs displacement $\Delta r=0$ $\mathrm{nm}$ ) and corresponding FT. (c) SLM simulation with $\sigma=20^{\circ}, \Delta r= \pm 1 \mathrm{~nm}$ and corresponding FT. (d) Radial averaged intensity $I(s)$ of the two SLM simulations. The gray profile is obtained from the FT of the SLM $\left(\sigma=0^{\circ}, \Delta r=0 \mathrm{~nm}\right)$, and the yellow one from the SLM $\left(\sigma=20^{\circ}, \Delta r= \pm 1\right.$ $\mathrm{nm}$ ). The $I(s)$ from the experimental FT of the TEM image is shown in purple. (e) CCFs at $s_{1,1}$ for the two SLM simulations compared to the FT of the experimental TEM image of the sample and color coded as in $\mathrm{d}$. ( $\mathrm{f}-\mathrm{i})$ SASA simulation obtained using the following parameters: (i) Electron energy: $30 \mathrm{keV}$, (ii) sample-detector distance: $230 \mathrm{~mm}$, (iii) sampling: $1000 \times 1000$ pixels, (iv) pixel size in the detector plane: $50 \mu \mathrm{m}$. (f) A single bunch of ordered ligands is selected for simulation. The radial intensity distribution shows a maximum at $s_{3}=1.45 \AA^{-1}$ for randomly oriented bunch of ligands and a maximum at $s_{4}=1.68 \AA^{-1}$ for oriented bunch of ligands. $(\mathrm{g})$ The diffraction pattern simulated from single bunch of ligands of a NP in preferred orientation exhibits two peaks at $s_{4}=1.68 \AA^{-1}$. (h) Diffraction pattern simulated from ligands on the NPs surfaces that are in preferred orientation and arranged into a hexagonal lattice exhibits six peaks at $s_{4}=1.68 \AA^{-1}$. (i) Diffraction pattern simulated from ordered ligands on the NPs surfaces that are in preferred orientation and arranged into a tetragonal lattice exhibits four peaks at $s_{4}=1.68 \AA^{-1}$.

membrane supporting the sample, and it is filtered in the analysis of the CCF (SI).

Experimental Diffraction Pattern. In Figure $2 \mathrm{a}-\mathrm{c}$, the results of the CCF analysis at the scattering vectors $s_{1}$ are reported. Such a distance in the reciprocal space corresponds to the planes of the supracrystal, rendered in Figure 2a. The spectrum of the intensity profile at $s_{1}$ (inset in panel b) is dominated by the frequency $\nu=2$ followed by $\nu=6$ and $\nu=4$, as shown in Figure 2b. The $\nu=2$ harmonic originates from the inherent slight astigmatism of the electron beam and the signal from the amorphous carbon of the substrate, as discussed in details in the SI. After filtering out $\nu=2$, a 4-fold modulation of the CCF is observed, see Figure $2 c$ (red curve). Next, after filtering out $\nu=2$ and $\nu=4$, a 6 -fold periodicity is retrieved in the CCF, as visible in Figure 2c (purple curve). At the reciprocal space distance $s_{3}$ corresponding to the arrangement of ligands in a superlattice on the NPs facets, rendered in Figure $2 \mathrm{~d}$, the CCF yields a clear 4-fold modulation after filtering out the $\nu=2$ Fourier component, as shown in Figure $2 \mathrm{f}$ (red curve). The absence of a 6 -fold arrangement at this distance is evident from the CCF (purple curve) shown in same figure. At $s_{4}$, corresponding to the distance between the carbon chains in the ligands, rendered in Figure 2g, the CCF shows a clear 4-fold modulation. When both the $\nu=2$ and $\nu=4$ frequencies are filtered out, a 6-fold CCF profile (Figure 2i, purple solid line) is observed.

Background Analysis. Among the retrieved periodicities, we determined which modulations originate solely from the scattering of electrons from the gold NPs and their ligands and which ones stem from the substrate. It is essential to verify that the diffraction intensity modulations in the experimental diffraction pattern are not coming from the TEM grid 
supporting the supracrystal or the substrate, but are a signature of the sample itself. Therefore, we applied the CCF and Fourier transform analysis to the experimental images acquired only from the substrate, without the sample. We compared the results from the dodecanethiol-coated NPs supracrystal with the ones obtained following the same acquisition process and data analysis on an amorphous carbon coated TEM copper grid and on an empty copper TEM grid. The static data from the supporting TEM grid in terms of electron flux, exposure time, and experimental geometry are identical to the ones reported for the acquisition of the electron diffraction pattern from the supracrystal, while those from the empty grid are obtained with much lower exposure time because the lower transmissivity of such a sample would cause the CCD saturation. The results, reported in Figure 3 and Figure 4, show that

(i) The 6-fold modulations of the intensity retrieved at $s_{1}$ and $s_{4}$ (Figure $2 \mathrm{c}$ and Figure $2 \mathrm{i}$ respectively, purple curves) and assigned to the local order within the supracrystal structure, are not found at $s_{1}$ and $s_{4}$ in the intensity of the diffraction pattern of the substrate. Thus, the 6-fold symmetries within the sample retrieved from the modulations in the CCFs at $s_{1}$ and $s_{4}$ can be safely attributed to the NPs supracrystal.

(ii) By comparison of the CCF at $s_{1}$ shown in Figure $2 \mathrm{c}$ (red curve) with that of the amorphous carbon shown in Figure $3 \mathrm{~b}$ (red curve), it turns out that both the supracrystal and the amorphous carbon show a similar 4-fold CCFs, that are also comparable in amplitude. Thus, we can attribute this 4-fold symmetry in the CCFs to the tetragonal arrangement of the amorphous carbon in the substrate.

(iii) The 4-fold symmetry retrieved at $s_{4}$ (Figure $2 \mathrm{i}$, red trace) from the diffraction pattern of the sample is not found at the same $s_{4}$ in the diffraction patterns, either of the amorphous carbon-coated or of the empty grid. Thus, this 4-fold modulation at $s_{4}$ solely originates from the NPs local arrangement in the supracrystal.

(iv) The 4-fold modulation retrieved at $s_{3}$ in the data from the amorphous carbon-coated grid (Figure $3 \mathrm{~d}$, red trace) confirms that the substrate contributes to a 4-fold modulation at $s_{3}$ (Figure $2 \mathrm{f}$, red trace).

(v) Both the NP supracrystal and the amorphous carbon samples show a strong and distinct 2-fold modulation at $s_{1}$, while the diffraction pattern of the empty TEM grid was overexposed at those $s$. The slight astigmatism of the beam is known from the original characterization of our experimental setup $^{21}$ and would also contribute to a 2 -fold modulation in the CCF.

The results of this analysis are summarized in Table 1 , where we show that the modulations of the CCF relative to the NPs arrangement in the supracrystal $\left(s_{1}\right)$ and the distance between chains of carbon atoms in ordered ligands $\left(s_{4}\right)$ can be solely attributed to the sample properties and not to either the amorphous carbon substrate (Figure 3 ) or the copper support grid itself (Figure 4).

Simulated Diffraction Patterns. Sphere Lattice Model and Superposition of Atomic Scattering Amplitude Simulations. To assign and explain the symmetries observed in the diffraction pattern to the corresponding real space objects, a series of simulations was carried out. Figure 5a displays the diffraction pattern simulated as the squared amplitude of the FT of the TEM image of the sample (Figure 1a), covering the scattering vector range up to a value of $0.4 \AA^{-1}$. To mimic the distribution of the NPs in the supracrystal we used the sphere lattice model (SLM) simulation, where the NPs are represented by a sphere, ${ }^{32}$ and their arrangement is governed by two parameters: NP diameter and deviation from the position in a perfect hexagonal lattice.

Two sample distributions simulated by the SLM at different disordering conditions are shown in Figure $5 b$ and $c$, with the corresponding Fourier transforms (FTs). The radial average intensity has been calculated as follows:

$$
I(s)=\frac{1}{2 \pi} \int I(s, \varphi) \mathrm{d} \varphi
$$

The radial intensity profiles for the two simulated and the experimental TEM-derived diffraction patterns are displayed in Figure $5 \mathrm{~d}$. The parameters of the SLM model were varied until the best match between the position of the peaks in the simulation and the experimental curve was achieved. The best match was found to be for a sphere diameter of $5.7 \mathrm{~nm}$, and a hexagonal arrangement with a core-to-core distance of $7.6 \mathrm{~nm}$. These parameters give the distance $d_{1}=6.6 \mathrm{~nm}$ between the planes of crystallographically arranged NPs in the supracrystal (upper inset of Figure 1a). The real space distances, $d_{1}$ and $d_{2}$, are introduced in the upper and lower panels of Figure 1a, respectively.

In Figure 5e, we show the CCF curves of the intensity distribution at a scattering vector $s$ corresponding to the first order of diffraction from the planes with spacing $\left.d_{1} s_{1,1}\right)$. The three CCFs show a 6-fold modulation, confirming the hexagonal arrangement of the NPs in the supracrystal. In the experimental electron diffraction pattern (Figure 1c, to be distinguished from the FT of the TEM image), the diffraction at $s_{1,1}$ is overexposed by the central beam. However, the higher diffraction orders of $d_{1}$ can be detected at higher $s$ values. For this reason, the analysis at $s_{1}\left(d_{1}=6.6 \mathrm{~nm}\right)$ in the experimental electron diffraction pattern was carried out at the seventh diffraction order $s_{1,7}\left(s_{1,7}=n \times 2 \pi / d_{1}=7 \times 2 \pi / 6.6 \mathrm{~nm}=0.66\right.$ $\left.\AA^{-1}\right)$, as marked in Figure 1c. Further discussion on this point is provided in the SI. The 6-fold CCF obtained for the experimentally measured intensity at $s_{1,7}$ (Figure 2c, purple curve) agrees well with the 6-fold CCFs obtained from simulated diffraction patterns (Figure 5e) for the lower diffraction orders of $s_{1}$. Thus, different diffraction orders from the same real-space object show an identical CCF periodicity. The simulations, the FT of the TEM image, and the small angle diffraction data all confirm that the NPs are arranged into a hexagonal lattice. Furthermore, the comparison of the SLM simulations to the experimental data allows us to quantitatively estimate the disorder in the crystal. The 4-fold modulation in the CCF retrieved from experimental intensity at $s_{1}$, and displayed in Figure 2c (red curve), is observed both in the sample with gold NPs and in an empty amorphous carbon coated grid. Therefore, the interpretation of this 4-fold symmetry cannot be done with certainty as such a 4-fold modulation could originate either from the amorphous carbon or from an inhomogenity in the supracrystal having tetragonally distorted domains.

The shorter distances, $d_{3}$ and $d_{4}$, are related to the atomic arrangement of gold cores and ligands. The simulated diffraction pattern at these distances is obtained by the superposition of atomic scattering amplitudes (SASA) from individual atoms in the NPs, where the shape of the NPs and the orientation of the ligands on their surface are modeled according to previous reports. ${ }^{32-34}$ It is worth noting that only an ordered structure gives rise to a characteristic peak in the diffraction pattern. For example, the wave scattered by gauche 

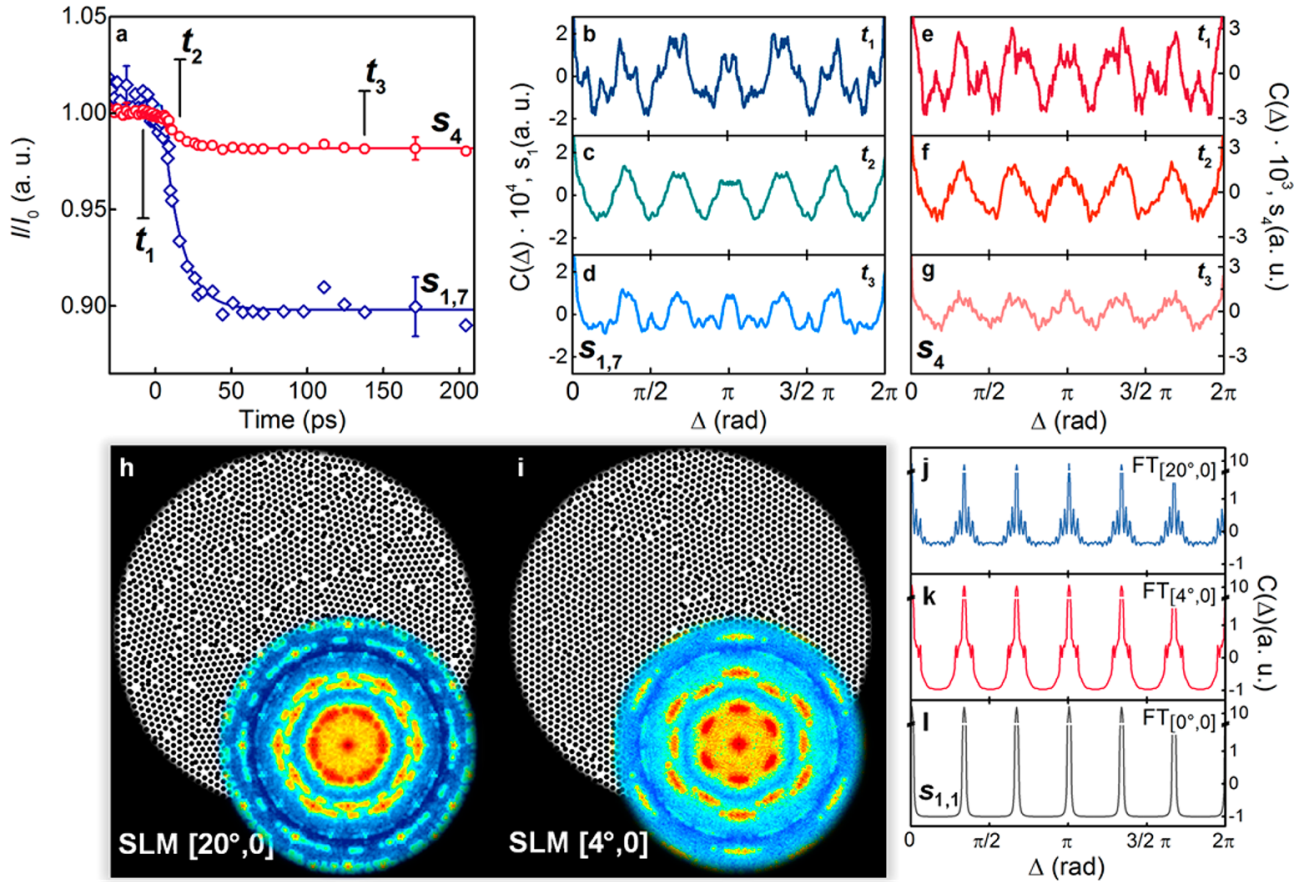

Figure 6. Small-angle ultrafast electron diffraction of dodecanethiol-capped gold NPs. (a) Dynamics of the radial intensity at $s_{1,7}$ (diamonds, blue trace) and $s_{4}$ (circles, red trace). Each intensity data set is normalized to its average value before time zero $\left(I_{0}\right)$ and it has been fitted to a monoexponential function. ( $\mathrm{b}-\mathrm{g})$ CCFs at $s_{1,7}(\mathrm{~b}-\mathrm{d})$ and $s_{4}(\mathrm{e}-\mathrm{g})$ at three time delays $\left(t_{1}, t_{2}, t_{3}\right)$. The "preferential orientation" evidenced at negative times $\left(t_{1}\right.$, panels $\left.\mathrm{b}, \mathrm{e}\right)$ is preserved upon photoexcitation. In fact, the 6-fold symmetry in the CCF is clearly preserved shortly after $\left(t_{2}\right.$, panels $\mathrm{c}, \mathrm{f})$ and more than $100 \mathrm{ps}$ after time-zero $\left(t_{3}\right.$, panels $\left.\mathrm{d}, \mathrm{g}\right)$, despite the photoinduced disorder, which is reflected in a decrease in the CCF amplitude upon photoexcitation. (h) SLM simulation with $\sigma=20^{\circ}, \Delta r=0 \mathrm{~nm}$ and corresponding FT. (i) SLM simulation with $\sigma=4^{\circ}, \Delta r=0 \mathrm{~nm}$ and corresponding FT. (j-1) CCFs at $s_{1,1}$ for the SLM simulations $\left[20^{\circ}, 0\right]$ (blue), $\left[4^{\circ}, 0\right]$ (red) and for the perfect lattice model (gray) of Figure 5b.

ligands does not create a characteristic peak but contributes to the background intensity. On the other hand, a bunch of equally oriented trans ligands constitutes a lattice of rods, which can give rise to characteristic scattering peaks in the diffraction pattern. For this reason, a single bunch of trans ligands attached to a gold facet was isolated for simulation, as depicted in Figure 5f. To simulate all the possible orientations of the bunch of ligands, and thus all possible orientations of the gold facets, the bunch was randomly rotated around its center. At each bunch rotation, for each atom, its $x, y, z$ coordinates were assigned, and the complex-valued scattering amplitudes specific to each chemical element $(\mathrm{Au}, \mathrm{S}, \mathrm{C}, \mathrm{H})$ were calculated using the NIST library $^{35}$ for $30 \mathrm{keV}$ electrons. The results of the SASA simulation are reported in Figure $5 f-i$. The complex-valued waves scattered off each atom were superimposed in the farfield and the intensity of the total wave field provided the diffraction pattern. For each rotation of the bunch such a simulated diffraction pattern was created. One hundred random rotations were calculated, and the related simulated diffraction patterns were added together (incoherent addition). Because of the stronger contribution from the carbon atoms, and of their intense scattering amplitude in the forward direction, their signal dominates the resulting diffraction pattern.

Figure $5 \mathrm{f}$ shows a magnified region of the radial intensity distribution calculated with eq 3 . The $I(s)$ obtained from the simulated diffraction pattern of randomly oriented bunches has a maximum at $s_{3}=1.45 \AA^{-1}$ (Figure 5f, blue curve). The CCF analysis of the intensity at $s_{3}$ in the simulated diffraction pattern (not shown here) does not reveal any modulations, which is predictable, as the bunch of ordered trans ligands was randomly rotated. On the other hand, a 4-fold modulation is revealed at $s_{3}$ in the experimental diffraction pattern (Figure $2 \mathrm{f}$ ), which can be attributed to tetragonal arrangement of the amorphous carbon in the substrate.

Next, in the simulation, the bunch of trans ligands was controllably rotated and a specific orientation of ligands with respect to the electron beam was achieved, which gives the diffraction peak at $s_{4}$. This arrangement is depicted in Figure $5 \mathrm{f}$ and in Figure 5g and named "preferred orientation"; the electron beam hits the sample orthogonally to the figure plane. "Preferred orientation" refers to the NP orientation under a specific polar angle, whereas the orientation of the NPs in the sample plane is arbitrary. A single bunch of ligands creates two peaks in the diffraction pattern, as shown in Figure $5 \mathrm{~g}$. When the NPs are arranged in a hexagonal lattice, as shown in the corner of Figure 5h, where each NP is in "preferred orientation", there are six possible orientations of the NPs in the sample plane, resulting in three possible orientations of bunch of ligands and hence six peaks in the diffraction pattern, see Figure $5 \mathrm{~h}$. When the NPs are arranged in a tetragonal lattice, as shown in the corner of Figure 5i, with each NP in "preferred orientation", there are two possible orthogonal orientations of bunch of ligands and hence four peaks in the diffraction pattern, see Figure 5i. Thus, this analysis demonstrates the presence of regions with tetragonal NPs arrangement within regions with hexagonal NPs arrangement in the supracrystal. The cross-validation between the simulations and the experimental data shows a good agreement: a 6-fold modulation was discovered at $s_{4}$ in the experimental diffraction pattern (Figure 2i), and no 6-fold modulations were found at $s_{3}$ (Figure 2f). 
Dynamics. Following the static characterization of the system, we studied the behavior of the NPs supracrystal under photoexcitation. In particular, we selected $s_{1}\left(s_{1,7}\right)$ to study the rearrangement of the NPs within the supracrystal, and we select $s_{4}$ to study the local rearrangement of the ligands. The radial intensity curves at the selected scattering vector $s$ have been retrieved following eq 3 and their amplitude as a function of the time delay is shown in Figure 6a.

The intensity traces as a function of time were normalized to their average value at negative times $\left(t<t_{0}\right)$ and fitted to a monoexponential curve. The energy deposited by the pump on the sample causes thermal disorder in the supracrystal, evidenced by a decrease of intensity at $s_{1}$ after the photoexcitation (blue curve, diamonds), with a time scale of $12 \pm 1$ ps. As a result, the supracrystal local hexagonal lattice arrangement is perturbed. The intensity at $s_{4}$ also decreases after the photoexcitation (red curve, circles), as a consequence of photoinduced thermal disorder $(10 \pm 1 \mathrm{ps})$. However, while the intensity of peak at $s_{1}$ drops by $10 \%$ the intensity of peak at $s_{4}$ drops by only $2 \%$, which means that although the NPs are slightly rearranging themselves, the ligands on their surfaces preserve the preferential arrangement shown before the photoexcitation. The recovery time for the rearranged system is beyond the probed time-scale range, i.e. longer than 220 ps after light exposure.

The evidenced dynamics is also confirmed by studying the CCF at both $s_{1}$ and $s_{4}$ at different time delays $\left(t_{1}, t_{2}, t_{3}\right)$, as shown in Figure $6 \mathrm{~b}-\mathrm{d}$, and Figure $6 \mathrm{e}-\mathrm{g}$. Here the CCFs were calculated as

$$
C(\Delta)=\langle I(s, \varphi) I(s, \varphi+\Delta)\rangle_{\varphi}-\langle I(s, \varphi)\rangle_{\varphi}^{2}
$$

where the normalization factor $\langle I(s, \varphi)\rangle_{\varphi}^{2}$ at the denominator was eliminated from the calculation in order to retrieve the absolute amplitude of the CCFs and to study its time dependence. The 6-fold modulations in the CCF for both $s_{1}$ and $s_{4}$ are reported at three selected time delays: before the photoexcitation $\left(t_{1}\right.$, Figure $6 \mathrm{~b}$ and $\left.\mathrm{e}\right)$, right after the photoexcitation $\left(t_{2}\right.$, Figure $6 \mathrm{c}$ and $\left.\mathrm{f}\right)$, and several picoseconds after photoexcitation ( $t_{3}$, Figure $6 \mathrm{~d}$ and $\mathrm{g}$ ). The amplitude decrease of the CCFs at both scattering vectors as a function of time is consistent with the dynamics in the system retrieved from the time traces of Figure 6a.

Remarkably, the 6-fold modulation of the CCF at both $s_{1}$ and $s_{4}$ is found to transiently become more evident as higher signalto-noise ratio (SNR) upon photoexcitation, despite decreasing in amplitude. As demonstrated by Latychevskaia et al., ${ }^{20}$ the amplitude of the CCF is proportional to the number of the domains, where the scatterers are organized into an ordered lattice. The experimental traces reported in Figure $6 \mathrm{~b}-\mathrm{d}\left(s_{1,7}\right)$ and Figure $6 \mathrm{e}-\mathrm{g}\left(s_{4}\right)$ show that the CCF amplitude is decreasing with time, indicating that the number of locally ordered regions is decreasing. However, the appearance of the peaks in the CCF is given by ordering in the orientation of the domains. A sample with equally oriented domains results in CCF peaks with high SNR, while a sample with more randomly oriented domains results in more noisy CCF peaks. This is demonstrated with the following SLM simulations. The perfect lattice arrangement of the NPs, Figure $5 \mathrm{~b}$, is modified by selecting round domains and rotating them by an angle $\eta$ which is Gaussian distributed with the mean $=0^{\circ}$ and standard deviation $\sigma=20^{\circ}$ (Figure 6h) and $\sigma=4^{\circ}$ (Figure 6i). The domains are selected to have a size of $60 \mathrm{~nm}$ and a center-to- center distance between domains of $80 \mathrm{~nm}$. For each simulation the corresponding FT is reported in Figure $6 \mathrm{~h}$ and i. The CCFs retrieved at $s_{1,1}$ for both FTs and for the perfect lattice arrangement are compared in Figure 6j-1. Upon increasing the degree of order in the supracrystal, by decreasing the rotation angle of each domain, peaks in the diffraction pattern become sharper. Consistently, the 6-fold CCF, which shows broad peaks characterized by the presence of satellites for the $\left[20^{\circ}, 0\right]$ case (Figure 6j), becomes sharper and more pronounced in the $\left[4^{\circ}, 0\right]$ case (Figure $6 \mathrm{k}$ ), all the way to the perfectly ordered lattice (shown in Figure 5b), in which the CCF consists of six sharp peaks (Figure 61). In our experiment, the SNR of the peaks in the CCF distribution for both $s_{1}$ (Figure $6 \mathrm{~b}-\mathrm{d}$ ) and $s_{4}$ (Figure $6 \mathrm{e}-\mathrm{g}$ ) reaches its maximum at $t_{2}$, after the photoexcitation, showing that the local rearrangement is triggered simultaneously for both the supracrystal and the ligands. The transient increase in the SNR of the CCF evidence that the system reaches some state with the maximal ordering of the domains but then it relaxes to a more favorable state, where some of NPs and the ligands are not ordered. Thus, while the photoexcitation process induces disordering within the supracrystal, the homogeneity of the distribution of the scattering improves, which in turn means that light excitation causes an overall disorder but also induces annealing of the grains in the sample.

Conclusions. In disordered elastic media, elastic forces of different origin, e.g., chemical, magnetic, or electrostatic, provide the binding necessary to create order. ${ }^{36}$ Their interplay with disorder, of thermal or quantum origin, gives rise to universal behaviors whose investigation has been mostly limited to the observation of frozen systems in static conditions. ${ }^{37,38}$ However, ordering and disordering phenomena are essentially dynamical. In our study, we demonstrate that it is now possible to determine the interplay between disorder and elastic forces with a spatial resolution that allows distinguishing every microscopic constituent of the system and a temporal resolution in the time scale of their motions.

\section{ASSOCIATED CONTENT}

\section{S Supporting Information}

The Supporting Information is available free of charge on the ACS Publications website at DOI: 10.1021/acs.nanolett.6b00355.

Spheres lattice model (SLM) simulations; superposition of atomic scattering amplitude (SASA) simulations and arrangement of ligands on the NP surface; crystallographic arrangement of NPs in the supracrystal: diffraction and angular cross-correlation functions at $s_{1}$ for higher diffraction orders; beam astigmatism and stability of the properties of the electron beam; analysis of the Fourier components as a function of $s$-vector coordinate; cross-validation of the absence of sample damaging (PDF)

\section{AUTHOR INFORMATION}

\section{Corresponding Author}

*Prof. Fabrizio Carbone, Laboratory for Ultrafast Microscopy and Electron Scattering (LUMES). Faculty of Basic Sciences (SB), École Polytechnique Fédérale de Lausanne (EPFL), EPFL Campus, CH H2 595, Station 6, CH-1015 Lausanne. Switzerland. E-mail: fabrizio.carbone@epfl.ch. Phone: +41 21 6930562. 


\section{Present Addresses}

G.F.M.: JILA, University of Colorado, Boulder, CO 803090440 .

J.R.: CIC biomaGUNE, Paseo de Miramón 182C, 20009

Donostia-San Sebastian, Spain.

J.R.: Ikerbasque, Basque Foundation for Science, 48011 Bilbao, Spain.

\section{Author Contributions}

F.C. and F.S. designed research; G.F.M. and F.P. performed research; G.F.M. and T.L. analyzed data; G.F.M., T.L., and F.C. wrote the paper; J.R. prepared the samples.

\section{Notes}

The authors declare no competing financial interest.

\section{ACKNOWLEDGMENTS}

The experimental work was funded by the Swiss National Science Foundation (SNSF) through the grant no. PP00P2128269/1. The authors acknowledge B. Patterson and A. Al Haddad for useful discussions. The authors declare no competing financial interest.

\section{REFERENCES}

(1) Kim, Y.; Zhu, J.; Yeom, B.; Di Prima, M.; Su, X.; Kim, J.-G.; Yoo, S. J.; Uher, C.; Kotov, N. A. Nature 2013, 500, 59-63.

(2) Pileni, M.-P. Acc. Chem. Res. 2007, 40, 685-693.

(3) Talapin, D. V.; Lee, J.-S.; Kovalenko, M. V.; Shevchenko, E. V. Chem. Rev. 2010, 110, 389-458.

(4) Feldheim, D. L.; Foss, C. A., Jr. Metal nanoparticles, synthesis, characterization and applications; CRC Press: New York, NY, 2001.

(5) Schmid, G. Nanoparticles: from theory to application; Wiley-VCH: Weinheim, Germany, 2006.

(6) Bishop, K. J. M.; Wilmer, C. E.; Soh, S.; Grzybowski, B. A. Small 2009, 5, 1600-1630.

(7) Nakanishi, H.; Walker, D. A.; Bishop, K. J. M.; Wesson, P. J.; Yan, Y.; Soh, S.; Swaminathan, S.; Grzybowski, B. A. Nat. Nanotechnol. 2011, 6, 740-746.

(8) De, M.; Ghosh, P. S.; Rotello, V. M. Adv. Mater. 2008, 20, 42254241.

(9) Schäfer, S.; Liang, W.; Zewail, A. H. Chem. Phys. Lett. 2011, 515, 278-282.

(10) Ernstorfer, R.; Harb, M.; Hebeisen, C. T.; Sciaini, G.; Dartigalongue, T.; Miller, R. J. D. Science 2009, 323, 1033-1037.

(11) Chen, J.; Chen, W.-K.; Tang, J.; Rentzepis, P. M. Proc. Natl. Acad. Sci. U. S. A. 2011, 108, 18887-18892.

(12) Chen, J.; Chen, W.-K.; Rentzepis, P. M. J. Appl. Phys. 2011, 109, 113522.

(13) Ruan, C.-Y.; Murooka, Y.; Raman, R. K.; Murdick, R. A. Nano Lett. 2007, 7, 1290-1296.

(14) Clark, J. N.; Beitra, L.; Xiong, G.; Higginbotham, A.; Fritz, D. M.; Lemke, H. T.; Zhu, D.; Chollet, M.; Williams, G. J.; Messerschmidt, M.; Abbey, B. R.; Harder, J.; Korsunsky, A. M.; Wark, J. S.; Robinson, I. K. Science 2013, 341, 56-59.

(15) Carbone, F.; Musumeci, P.; Luiten, O. J.; Hebert, C. Chem. Phys. 2012, 392, 1-9.

(16) Piazza, L.; Musumeci, P.; Luiten, O. J.; Carbone, F. Micron 2014, 63, 40-46.

(17) Spence, J. C. H.; Weierstall, U.; Howells, M. Ultramicroscopy 2004, 101, 149-152.

(18) Wochner, P.; Gutt, C.; Autenrieth, T.; Demmer, T.; Bugaev, V.; Díaz Ortiz, A.; Duri, A.; Zontone, F.; Grübel, G.; Dosch, H. Proc. Natl. Acad. Sci. U. S. A. 2009, 106, 11511-11514.

(19) Wochner, P.; Castro-Colin, M.; Bogle, S. N.; Bugaev, V. N. Int. J. Mater. Res. 2011, 102, 874-888.

(20) Latychevskaia, T.; Mancini, G. F.; Carbone, F. Sci. Rep. 2015, 5, 16573.
(21) Mancini, G. F.; Mansart, B.; Pagano, S.; van der Geer, S. B.; de Loos, M. J.; Carbone, F. Nucl. Instrum. Methods Phys. Res., Sect. A 2012, 691, 113-122.

(22) Ong, Q. K.; Reguera, J.; Silva, P. J.; Moglianetti, M.; Harkness, K.; Stellacci, F. ACS Nano 2013, 7, 8529-8539.

(23) Zheng, N.; Fan, J.; Stucky, G. D. J. Am. Chem. Soc. 2006, 128, $6550-6551$.

(24) Lee, Z.; Jeon, K.-J.; Dato, A.; Erni, R.; Richardson, T. J.; Frenklach, M.; Radmilovic, V. Nano Lett. 2009, 9, 3365-3369.

(25) Panthani, M. G.; Hessel, C. M.; Reid, D. K.; Casillas, G.; JoseYacaman, M.; Korgel, B. A. J. Phys. Chem. C 2012, 116, 22463-22468.

(26) Gunawan, A. A.; Chernomordik, B. D.; Plemmons, D. S.; Deng, D. D.; Aydil, E. S.; Mkhoyan, K. A. Chem. Mater. 2014, 26, 33283333.

(27) van Oudheusden, T.; Pasmans, P. L. E. M.; van der Geer, S. B.; de Loos, M. J.; van der Wiel, M. J.; Luiten, O. J. Phys. Rev. Lett. 2010, $105,264801$.

(28) Kiewiet, F. B.; Kemper, A. H.; Luiten, O. J.; Brussaard, G. J. H.; van der Wiel, M. J. Nucl. Instrum. Methods Phys. Res., Sect. A 2002, 484, 619-624.

(29) Musumeci, P.; Moody, J. T.; Scoby, C. M.; Gutierrez, M. S.; Westfall, M. Appl. Phys. Lett. 2010, 97, 063502.

(30) Altarelli, M.; Kurta, R. P.; Vartanyants, I. A. Phys. Rev. B: Condens. Matter Mater. Phys. 2010, 82, 104207.

(31) Kurta, R.; Altarelli, M.; Weckert, E.; Vartanyants, I. A. Phys. Rev. B: Condens. Matter Mater. Phys. 2012, 85, 184204.

(32) Badia, A.; Singh, S.; Demers, L.; Cuccia, L.; Brown, R.; Lennox, R. B. Chem. - Eur. J. 1996, 2, 359-363.

(33) Whetten, R. L.; Khoury, J. T.; Alvarez, M. M.; Murthy, S.; Vezmar, I.; Wang, Z. L.; Stephens, P. W.; Cleveland, C. L.; Luedtke, W. D.; Landman, U. Adv. Mater. 1996, 8, 428-433.

(34) Badia, A.; Cuccia, L.; Demers, L.; Morin, F.; Lennox, R. B. J. Am. Chem. Soc. 1997, 119, 2682-2692.

(35) NIST, electron elastic-scattering cross-section database 2000, NIST Standard Reference Database 71. http://www.nist.gov/srd/ nist64.cfm.

(36) Giamarchi, T. in Encyclopedia of Complexity and Systems Science; Springer: New York, NY, 2009; pp 2019-2038.

(37) Giamarchi, T.; Le Doussal, P. Phys. Rev. B: Condens. Matter Mater. Phys. 1995, 52, 1242-1270.

(38) Klein, T.; Joumard, I.; Blanchard, S.; Marcus, J.; Cubitt, R.; Giamarchi, T.; Le Doussal, P. Nature 2001, 413, 404-406. 